\title{
Experimental analysis of non-periodic sound regimes in flute-like musical instrument
}

\author{
Soizic Terrien \\ Laboratoire d'Acoustique de l'Université du Mans (LAUM), UMR 6613, \\ Institut d'Acoustique - Graduate School (IA-GS), CNRS, Le Mans Université, France \\ Christophe Vergez \\ Aix Marseille Univ, CNRS, Centrale Marseille, LMA UMR7031, \\ 4 impasse Nikola Tesla, 13013, Marseille, France \\ Patricio de la Cuadra \\ Escuela de Ingeniera-Instituto de Msica, Pontificia Universidad Catlica de Chile, Santiago. \\ Benoît Fabre \\ Sorbonne Université, UMR 7190, LAM- Institut d'Alembert, 75252 Paris France \\ Self-sustained musical instruments are complex nonlinear dynamical systems which are \\ known to produce a wealth of dynamical regimes. This includes different kinds of non \\ periodic sounds, which are either played on purpose or avoided depending on the cultural \\ and musical context. We investigate non-periodic sounds produced by two types of flute-like \\ instruments, namely an alto recorder and traditional pan-like flutes from Central Chile. We \\ adopt a nonlinear dynamics point of view to characterize the multiphonics produced by the \\ alto recorder and the sonidos rajados produced by the Chilean flutes. Our results unveil \\ the common quasiperiodic nature of the two types of sound regimes, and suggest that they \\ result from a similar physical sound production mechanism. This paves the way for a better \\ control of non periodic sound regimes by the instrument makers.
}




\section{INTRODUCTION AND PROBLEM STATEMENT}

Flute-like musical instruments have been shown, both experimentally and theoretically, to produce a wealth of different sound regimes [1-3]. Most studies focus on periodic regimes which generally correspond to the desired behavior of the instrument. Nonperiodic regimes have been investigated both in flute-like instruments and in other wind instruments [4-11]; however, both their precise nature and the underlying physical mechanisms of sound production remain poorly understood.

In the context of western classical music, non periodic sounds often result from a defect of the instrument, or from a lack of control of the musician. This is, for example, the case of "rolling sounds" in flute-like instruments which instrument makers try to avoid [12]. On the other hand, some non-periodic sounds (often referred to as multiphonics) are also played on purpose, in particular in jazz and contemporary music.

Non periodic sounds are also widely used in other cultural contexts. Flautas de chinos are pre-hispanic flutes from central Chile. These are still played in traditional religious processions in which the participants aim at "filling the space, time and frequency spectrum in order to achieve a kind of trance" [11, 13]. Approximately 20 instruments of different sizes are traditionally played simultaneously. Each instrument produces a single "note", namely a loud non-periodic sound referred to as sonido rajado (literally "torned sound").

In this article, we focus on the characteristics of non periodic sounds produced by these two families of flute-like instruments: the multiphonics of recorders on the one hand, and the sonidos rajados of flautas de chinos on the other hand.

The sonidos rajados produced by chilean flutes have been characterized in terms of spectral content and modulation amplitude and frequency $[8,13]$. Particular features of these sounds include high-order partials with high energy levels, as well as a strong beating component called ganseo [13]. In parallel, the spectral content of multiphonic sound regimes produced by recorders has been characterized in several studies $[6,7,14]$. The spectral characteristics of both kind of sounds have been compared, and the physical mechanism responsible for the emergence of multiphonics in recorders has been investigated [5]. In particular, it has been suggested that multiphonics result from a beating phenomenon, due to a slight detuning between a high-order resonance frequency of the instrument and one of the harmonics of the periodic sound normally produced by the recorder. However, no conclusions were drawn neither on the production mechanism of sonidos rajados, nor on the exact nature of both nonperiodic regimes. 


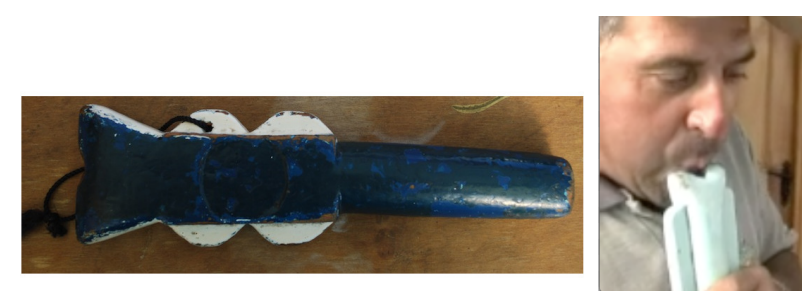

FIG. 1. Two pan-like flutes from Central Chile (flautas de chinos): a Catarra (left) and a Puntera played by a musician (right).

We aim here at determining the precise nature of multiphonics of recorders and sonidos rajados of flautas de chinos. In particular, we wonder if these two kind of regimes share, or not, a same quasiperiodic, intermittent or chaotic nature. This is an essential first step in the attempt to understand the production mechanism of non periodic sounds in flute-like instruments. Understanding whether or not the sonidos rajados and the multiphonics result from a similar physical mechanism would be particularly valuable both from a fundamental point of view and in the context of applications to instrument making. In particular, this would allow to investigate systematically the influence of making and playing parameters on the emergence of non periodic regimes.

In section 2, we describe the considered Chilean pan-like flutes and recorder in more details. In section 3 , the properties of the two types of non-periodic sounds are investigated using advanced methods dedicated to the analysis of nonlinear time series. This allows to conclude on the quasiperiodic nature of both types of sounds. Section 4 provides preliminary observations on the underlying mechanism for the emergence of the quasiperiodic regimes through the comparison between the resonance frequencies of the recorder and the base frequencies involved in the quasiperiodic regimes. Finally, we conclude and provide some perspectives for future work.

\section{DESCRIPTION OF THE INSTRUMENTS AND EXPERIMENTAL CONDITIONS}

Figure 1 shows two different chilean flutes, referred to as Catarra and Puntera. They differ in size, but feature a common geometry that is characteristics of flautas de chinos. As shown schematically in Figure 2, the resonator consists of two mostly cylindrical pipes in series, with a closed end and no lateral toneholes. The dimensions of both pipes vary from an instrument to the other, but the two pipes of a given instrument are generally of similar length and the ratio between the diameters of the first (on the musician's side) and the second pipe is approximately $1.6[8,11]$. The Puntera flute has a first pipe of $310 \mathrm{~mm}$ in length and $18 \mathrm{~mm}$ in (internal) diameter, and a second pipe closed at its end of $300 \mathrm{~mm}$ in length and $11 \mathrm{~mm}$ in diameter. The Catarra is 

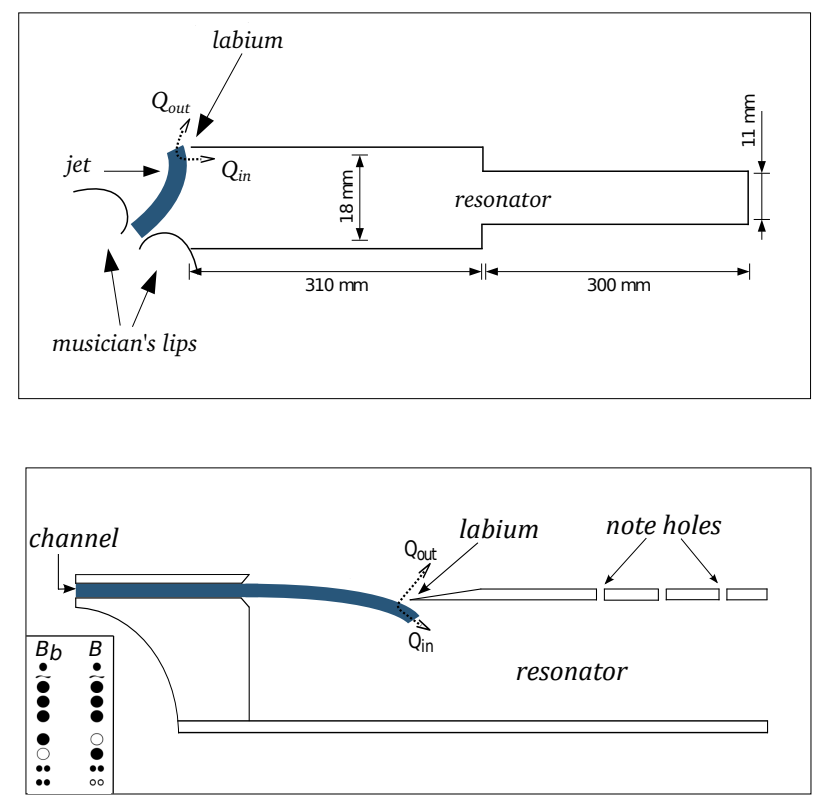

FIG. 2. Schematic representation of a Puntera flauta de chinos (top) and a recorder (bottom). The air jet oscillating around the labium is represented in blue (color online). $Q_{\text {in }}$ and $Q_{\text {out }}$ are the fraction of the air flow going inside and outside the resonator (respectively) within an oscillation period of the jet. The inset at the bottom left shows schematic representations of the two fingerings (combinations of open and closed note holes) considered in the article for the recorder.

significantly smaller, with two pipes which are $111 \mathrm{~mm}$ and $124 \mathrm{~mm}$ long, and $17 \mathrm{~mm}$ and $10.5 \mathrm{~mm}$ wide, respectively.

The resonator of recorders, on the contrary, consists of a complex but continous conic-shaped pipe with an open end, as shown in Figure 2. We consider here an alto Zen-On recorder (model Bressan) with ten lateral toneholes, whose geometry has been characterized in details by Lyons $[15]$.

The playing technique of the chilean flutes also differs significantly from that of recorders. Flautas de chinos are pan-like flutes: the musician blows on the edge of the opened end of the resonator, as shown in Figures 1 (right) and 2 (top). In the case of a recorder on the other hand, the musician blows into a narrow channel, as shown in Figure 2 (bottom). This induces an air jet at the other end of the channel, which propagates through a window and subsequently interacts with a sharp edge of the instrument (the labium).

In spite of these differences, the sound production in recorders and flautas de chinos results from similar physical mechanisms, which are characteristics of flute-like musical instruments; see for example [16]. When the musician blows in the instrument, an air jet is created either at the channel exit in the case of recorders (as described above) or at the end of the musician's lips for the chilean flutes. The air jet is intrisically unstable and therefore starts to oscillate around the labium. 
The air jet oscillations result in a flow injection alternatively inside and outside the instrument pipe: this constitutes a dipolar pressure source for the resonator, which consists of the air column contained in the instrument. Acoustic waves thus created propagate in the resonator and this acoustic field perturbs back the jet at the channel exit, thus sustaining the oscillations of the air jet and, as such, the sound production. Self-sustained oscillations in flute-like instruments thus result from a nonlinear interaction between an oscillating air jet and a passive acoustic resonator.

In this article, we consider two different non-periodic sounds (sonidos rajados) produced by the two chilean flutes described above (Catarra and Puntera). To the best of our knowledge, the playing technique of Pan-like flutes has not been reproduced successfully with an artifical mouth. The instruments were thus played by an experienced player. The pressure $P_{m}$ in the musician's mouth was measured through a small pipe sealed to a manometer and the radiated sound was recorded with a sampling frequency $f_{s}=44.1 \mathrm{kHz}$. As a consequence, the measured signals are non-stationary, with significant variations of the mouth pressure. Moreover, because the chilean flutes are played for high values of the mouth pressure (between $P_{m}=886 \mathrm{~Pa}$ and $P_{m}=1546 \mathrm{~Pa}$ ) and air flow, the sound can only be sustained for about one second by the musician. As such, only short signals are available.

In parallel, we consider two different non-periodic sounds (multiphonics) produced by an alto Zen-On recorder [15]. These multiphonics are produced using two different fingerings (i.e. two different combinations of open and closed note holes of the resonator) which are generally used to produce a $\mathrm{B}$ flat (noted $\mathrm{B}_{b}$ hereafter) and a $\mathrm{B}$, respectively. These fingerings are represented schematically in the inset in Figure 2. The recorder was played by a pressure-controlled artificial mouth [17], which allows to play the instrument without musician. The playing parameters related to the musician (in particular the mouth pressure $P_{m}$ ) are controlled precisely in a continuous manner. As a result, the experimental measurements are highly reproducible, and the measured signals are stationary. During the experiment, the (controlled) mouth pressure, the radiated sound and the acoustic pressure signal inside the resonator (under the labium) are measured simultaneously with a sampling frequency $f_{s}=20 \mathrm{kHz}$. For the two considered fingerings, the multiphonics are observed for higher values of the mouth pressure compared to those for which the first register (i.e. the periodic regime corresponding to notes $\mathrm{B}_{b}$ and $\mathrm{B}$ ) is obtained. In particular, the multiphonics were measured here for $P_{m}=650 \mathrm{~Pa}$ and $P_{m}=560 \mathrm{~Pa}$ for the $\mathrm{B}_{b}$ and B fingerings, respectively. On this instrument and with the artificial mouth, we observe the periodic regimes corresponding to the notes $\mathrm{B}_{b}$ and $\mathrm{B}$ for values of the the mouth pressure under $P_{m}=505 \mathrm{~Pa}$ and under $P_{m}=485$ $\mathrm{Pa}$, respectively [18]. 

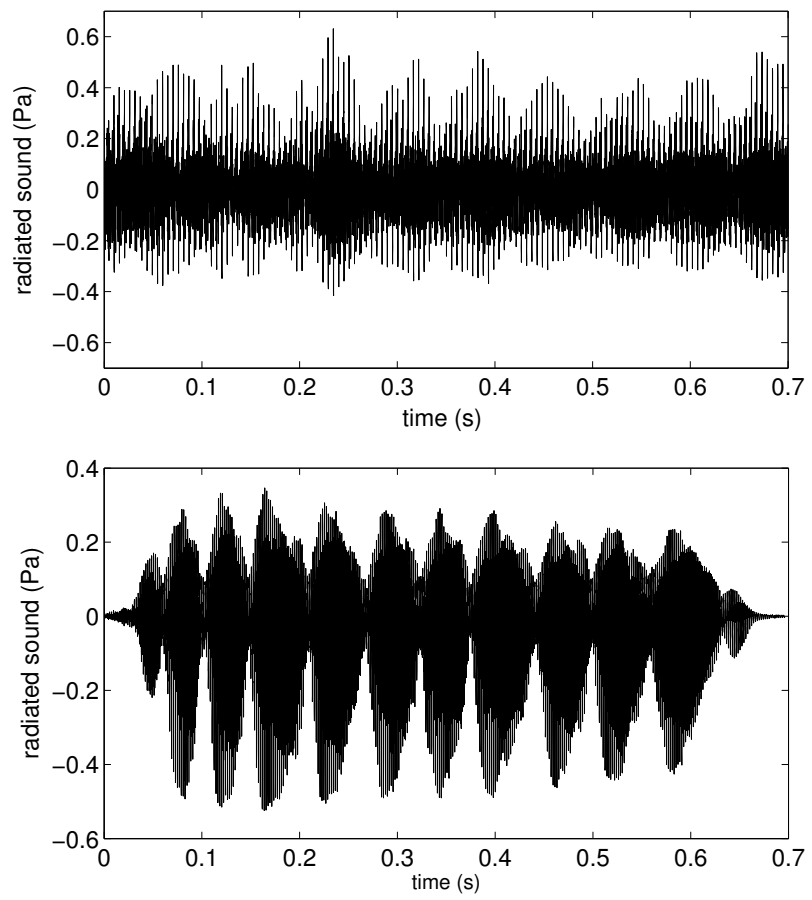

FIG. 3. Radiated sound produced by two flautas de chinos of different size: a Puntera (top) and a Catarra (bottom). Both instruments were played by an experienced flute player. The pressure in the musician's mouth was between $886 \mathrm{~Pa}$ and $1546 \mathrm{~Pa}$ for the Puntera, and between $1255 \mathrm{~Pa}$ and $1266 \mathrm{~Pa}$ for the Catarra.

\section{ANALYSIS OF EXPERIMENTALLY-OBSERVED NON PERIODIC REGIMES}

\section{A. Time series}

Figure 3 shows the time series associated with the (radiated) non-periodic sounds (sonidos rajados) produced by the two chilean flutes (Puntera and Catarra) described in section II when played by a musician [19] [20]. The pressure $P_{m}$ measured in the musician's mouth varies between $886 \mathrm{~Pa}$ and $1546 \mathrm{~Pa}$ for the Puntera, and between $1255 \mathrm{~Pa}$ and $1266 \mathrm{~Pa}$ in the case of the Catarra. The time series in Figure 3 show a deep modulation of the amplitude at a frequency close to $20 \mathrm{~Hz}$, corresponding to the strong beating component which is characteristics of the sounds produced by these instruments [13].

Figure 4 shows the time series corresponding to the two radiated non periodic sounds (multiphonics) produced by an alto recorder played by a pressure-controlled artifical mouth for the $B_{b}$ and $B$ fingerings as described above [21]. The controlled pressure in the artificial mouth is set to $P_{m}=650 \mathrm{~Pa}$ and $P_{m}=560 \mathrm{~Pa}$ for the $\mathrm{Bb}$ and $\mathrm{B}$ fingerings, respectively. Compared to the sonidos rajados in Figure 3, the time series in Figure 4 highlight an amplitude modulation at a much larger 

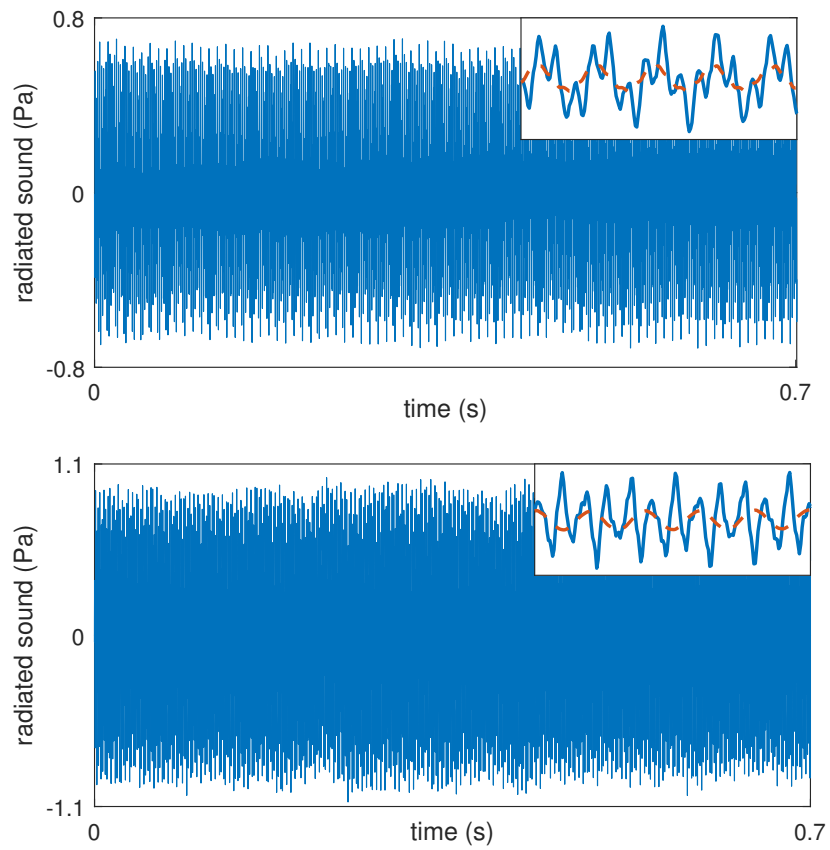

FIG. 4. Times series corresponding to the sounds radiated by a alto Zen-On recorder for a $\mathrm{B}_{b}$ fingering with a mouth pressure $P_{m}=650 \mathrm{~Pa}$ (top) and for a B fingering, with a mouth pressure $P_{m}=560 \mathrm{~Pa}$ (bottom). The instrument was played by a pressure-controlled artificial mouth. The insets show zooms on the first 0.01 seconds of the signals (solid blue line), and show a comparison with the waveform of the periodic sounds (dashed red line) corresponding to notes $\mathrm{B}_{b}$ (top) and $\mathrm{B}$ (bottom) obtained for the same fingerings but for lower values of $P_{m}(350 \mathrm{~Pa}$ and $400 \mathrm{~Pa}$, respectively).

(faster) modulation frequency. The amplitude and frequency of the modulation appear more clearly in the zooms shown in the insets of Figure 4. For sake of comparison, these insets also show (red curves) the waveforms of the two periodic sounds associated with notes $B_{b}$ and $B$. These are obtained with the pressure-controlled artificial mouth for the same fingerings as the multiphonics, but for lower values of the mouth pressure $\left(P_{m}=350 \mathrm{~Pa}\right.$ for the $B_{b}$ and $P_{m}=400 \mathrm{~Pa}$ for the B). The fundamental frequencies of these periodic sounds are $471 \mathrm{~Hz}$ and $504 \mathrm{~Hz}$, respectively.

We aim here at performing an in-depth analysis of these two kinds of non-periodic sounds and, in particular, at characterizing these regimes from a nonlinear dynamics point of view. Indeed, nonperiodic regimes include chaotic dynamics and quasiperiodic dynamics, but also nonstationary effects on periodic oscillations, due for example to noise and uncontrolled fluctuations of the control parameters (for example here the mouth pressure $P_{m}$ ). Chaos and quasiperiodicity correspond, mathematically, to well-known and precisely defined dynamics; see for example [22, 23] as entry points to the extensive literature. Although experimentally-observed chaotic regimes can look like noise both in the time and Fourier domains, they fundamentally differ from stochastic regimes as they result from fully deterministic systems, and no random process is involved [22]. In other words, 
the long term behaviour remains determined by the initial conditions of the system (although it is very sensitive to these initial conditions) [22]. On the other hand, quasiperiodic regimes correspond to oscillations with a finite number of rationally linearly independent frequencies $f_{1}, \ldots, f_{n}$, that is to say:

$$
a_{1} f_{1}+\cdots+a_{n} f_{n} \neq 0
$$

for any non-zero integers $\left(a_{1}, \ldots, a_{n}\right)$. Hereafter, these frequencies are referred to as base frequencies of the quasiperiodic regime. The spectrum of a quasiperiodic regime contains several peaks, at frequencies equal to linear combinations of the base frequencies [22, 24].

\section{B. Spectral analysis}

Figure 5 and 6 show the modulus of the spectra associated with the sonidos rajados produced by the two chilean flutes and with the multiphonics produced by the alto recorder, respectively. For the four different sounds (two sonidos rajados and two multiphonics), the spectra show a large number of peaks, at frequencies which are not all integer multiples of the frequency of the first partial. This confirms the non-periodic feature of both types of sounds. However, a Fourier analysis is not necessarily sufficient to conclude on the exact nature of such non-periodic sounds, and to distinguish for example a quasiperiodic regime from a chaotic regime [22]. It is particularly true here for the two sonidos rajados: because the signals are highly non-stationary, their spectra only provide a rough estimation of the oscillation frequencies for the considered (mean) value of the mouth pressure.

\section{Nature of sonidos rajados}

Different numerical methods allow one to distinguish a quasiperiodic signal from a chaotic signal. Classical approaches include the computation of Poincaré sections and the estimation of Lyapunov exponents $[22,24]$. However, such methods are not suitable for short, non-stationary signals. In contrast, the recurrence plots approach [25] allows to estimate the periodicity properties of a signal at different time scales and is both robust to noise and suitable for short signals.

The idea behind the recurrence plots analysis is to consider the trajectory described by a system in its phase-space, and to determine the return times after which the trajectory returns to 

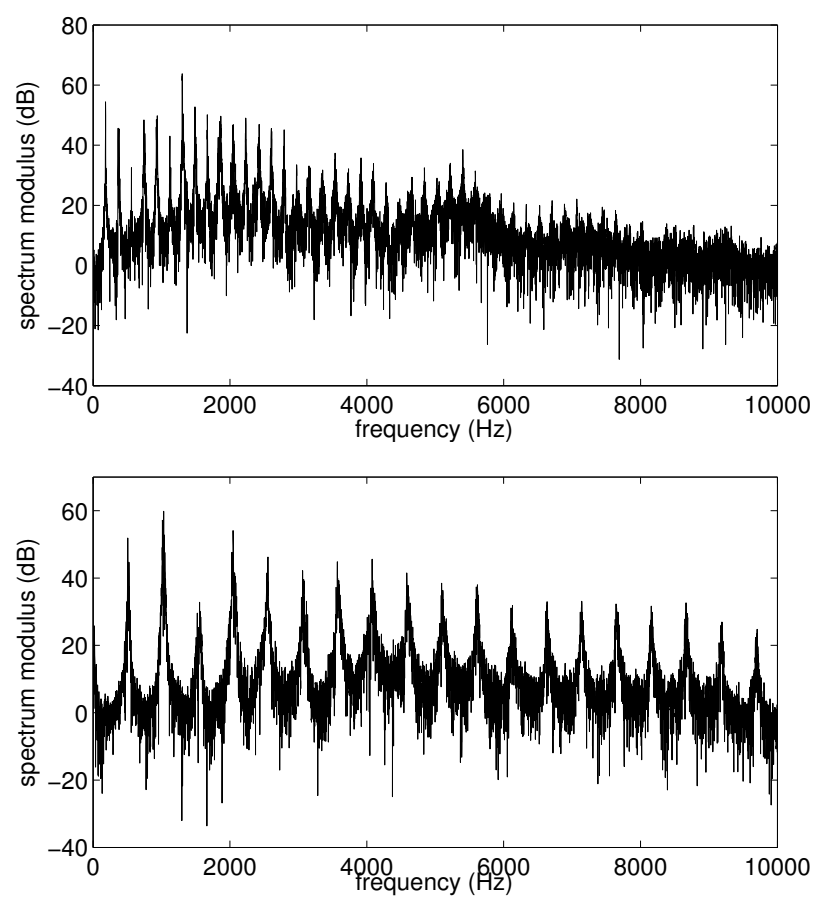

FIG. 5. Modulus of the spectra associated with the two sonidos rajados represented in Figure 3, produced by a Puntera flute (top) and a Catarra flute (bottom). The modulus is represented in $\mathrm{dB}$ (reference pressure $1 \mathrm{~Pa})$.
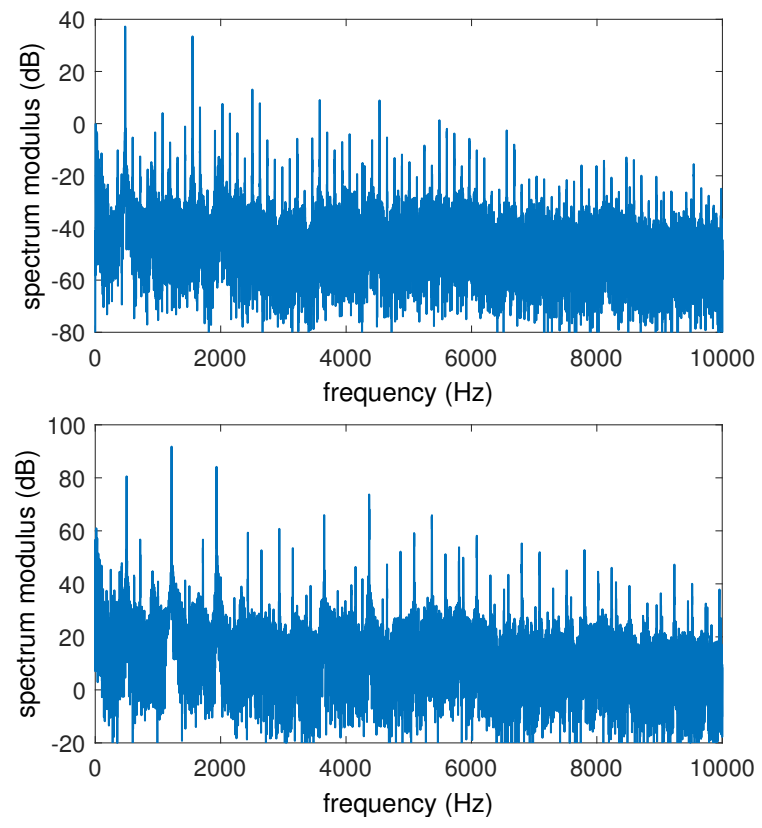

FIG. 6. Modulus of the spectra associated with the two multiphonics represented in Figure 4, produced by an alto recorder for $\mathrm{a}_{b}$ fingering with a mouth pressure of $650 \mathrm{~Pa}$ (top) and for a $\mathrm{B}$ fingering with a mouth pressure of $560 \mathrm{~Pa}$ (bottom). The modulus is represented in $\mathrm{dB}$ (reference pressure $1 \mathrm{~Pa}$ ). 
the neighborhood of one of its previous point. In the simple case of a noise-free periodic regime, this analysis would return a single return time associated with the period of the oscillation. In the case of experimental signals, one generally does not know the dimension of the phase space of the physical system. The method here consists in embedding the time series $s(t)$ in a reconstructed pseudo-phase space, whose dimensions are delayed versions $s(t-\tau), s(t-2 \tau) \ldots$ of the signal itself [22]. The delay is chosen as the first minimum of the auto-correlation of the signal $s(t)$, and the embedding dimension is determined following the method by Cao [26]. In this pseudo-phase space, the signal describes a trajectory. A small neighborhood is defined around each point of the trajectory, and the return times after which the trajectory comes back in this neighborhood are determined. Histograms are plotted, which represent the occurence of the different returns times. This provides insight on the periodicity properties of the considered time series [27]. This analysis is increasingly precise as more reference points are considered along the trajectory. This method, implemented in the toolbox Open TSTOOL [28], is used here to analyze the sonidos rajados represented in Figure 3. The corresponding histograms are represented in Figure 7. They both highlight three main return times: two of them are non-commensurate (hereafter, we call them base return times), and the third one is the sum of the two first ones. According to Slater's theorem [27], this is characteristic of quasiperiodic regimes. This can be understood as follows: in the phase space, a quasiperiodic regime corresponds to a trajectory lying on an invariant torus [24], i.e. an object which can be generated by the rotation of a circle around another circle. As such, a trajectory on a torus can be characterized, in the context of nonlinear dynamics, by two independent periods (or frequencies) associated with each rotation [22]. If the ratio of the two frequencies is rational, then there is a time interval after which the trajectory comes back exactly to a same point on the torus, and the motion is thus periodic (and often referred to as being "locked" on the torus [24]). On the other hand, if the ratio of the two frequencies is irrational, the motion is quasiperiodic. As such, a quasiperiodic regime features, by definition, two characteristic timescales, which are referred to as base frequencies or base return times in this article.

An important point here is that the dimension of the underlying physical system (and as such the dimension of the phase space) is unknown and can only be estimated [26]. As such, the dimension of the pseudo-phase space used for the return times analysis generally differs from the dimension of the phase-space of the physical system. This is particularly true here: flute-like instruments are delay systems, whose phase-space is of infinite dimension [29]. As such, one considers necessarily a projection of the trajectory in a low-dimensional pseudo-phase space. This explains why the return times obtained through this reconstruction do not necessarily correspond to the base frequencies 
observed in the spectra of the experimental signals. However, the main purpose of the return time analysis is to uncover the nature of the experimentally-observed regimes, and in particular to distinguish between quasiperiodic and chaotic dynamics.

In the ideal case of a noise-free quasiperiodic signal, the histograms represented in Figure 7 would only display peaks for three return times (the two base return times and the one corresponding to their sum). However, it has been demonstrated that noise can trigger additional peaks, for return times equal to linear combinations of the two base return times [27], which is observed here for both histograms. This can be understood as follows: in the pseudo phase space, small amounts of noise induce a dispersion of the trajectory points around the noise-free (ideal) trajectory. Consequently, some trajectory points which would, in the noise-free case, lie inside the neighborhood of a previous point of the trajectory are brought outside of this neighborhood under the effect of noise. This results in the non detection of thus missed returns. The importance of the phenomenon depends on both the amount of noise and the size of the considered neighborhood around each reference point. In the case of a missed return, the next return will therefore be detected for a larger value of time (thus resulting in the detection of a larger return time) at a linear combination of one of the base return times, due to the periodicity properties of the time series.

Overall, the return times analysis of the sonidos rajados, in Figure 7, clearly demonstrates the quasiperiodic nature of these sound regimes.

\section{Nature of recorder multiphonics}

Conversely to flautas de chinos, recorders can be studied experimentally using a pressure controlled artificial mouth [17]. This makes long, stationary sounds easy to measure, as shown in figure 4. For sake of consistency with the results presented on the chilean flutes, we first perform here a return time analysis. Figure 8 shows return times histograms corresponding to the two multiphonics represented in Figure 4. For both multiphonics, these highlight two non-commensurate base return times, as well as a third return time which is equal to the sum of the two base ones. Because we deal here with stationary signals with low noise level, the histograms only display these three peaks. This analysis thus clearly shows that multiphonics of recorders are quasiperiodic regimes.

The study of the Poincaré sections of the two multiphonic signals confirms their quasiperiodic nature. The Poincaré section of a time series corresponds to the transverve section of its trajectory in its phase space. As for the return times estimation, the signals are here embeded in a suitable pseudo-phase space [28]. Because quasiperiodic oscillations take place on an invariant torus, the 

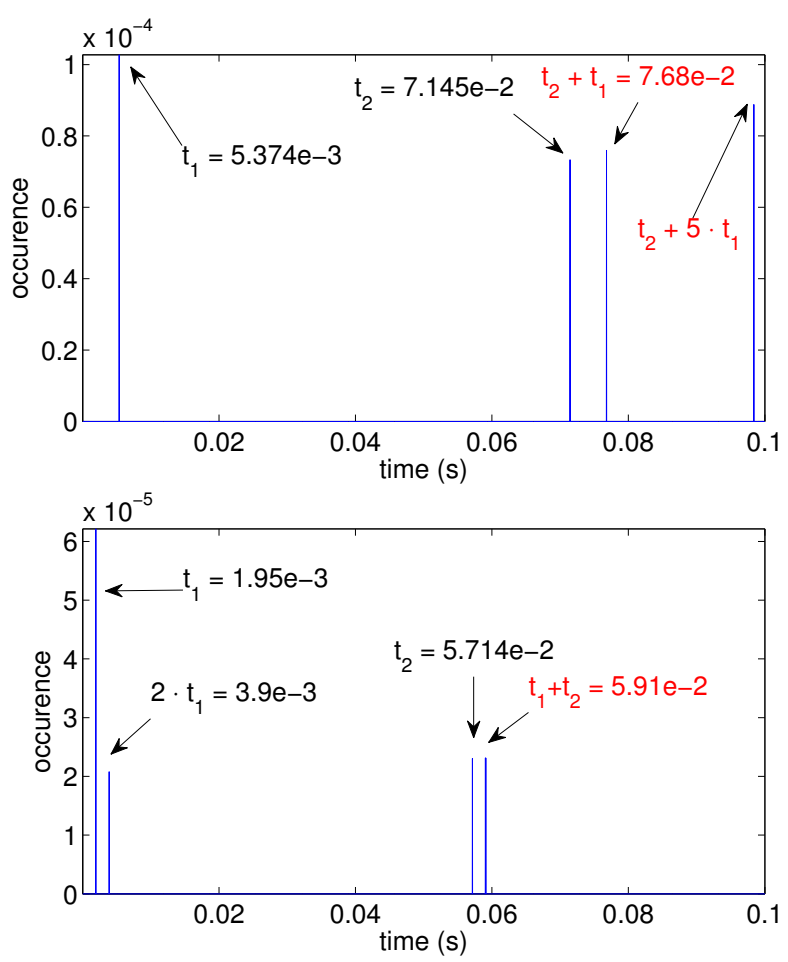

FIG. 7. Histograms of the return times associated with the sonidos rajados produced by a a Puntera chilean flute (top) and a Catarra chilean flute (bottom). The associated time series are represented in figure 3.

corresponding Poincaré section is a densely filled closed curve [24]. The Poincaré sections associated with both multiphonics, shown in Figure 9, are thus characteristics of quasiperiodic regimes. Importantly, these Poincaré sections were plotted from the acoustic pressure measured inside the recorder, under the labium (see [17] for the experimental device), as the internal field is less noisy than the radiated acoustic field.

\section{PRODUCTION MECHANISM OF QUASIPERIODIC SOUNDS: PRELIMINARY OBSERVATIONS}

The comparison between the base frequencies of quasiperiodic regimes and the resonance frequencies of the instrument can provide valuable insight on the underlying physical mechanism of (quasiperiodic) sound production [5]. In other blowing musical instruments, such as brass and reed instruments, the playing frequencies are related to the peaks of the input impedance of the resonator, which is in a close-open configuration. On the other hand, recorders and transverse flutes operate in an open-open resonator configuration, and the playing frequencies are close to the minima of the input impedance (i.e. the maxima of the input admittance). Furthermore, the radiation impedance at the open end has to be taken into account [30]. Experimental techniques have been 

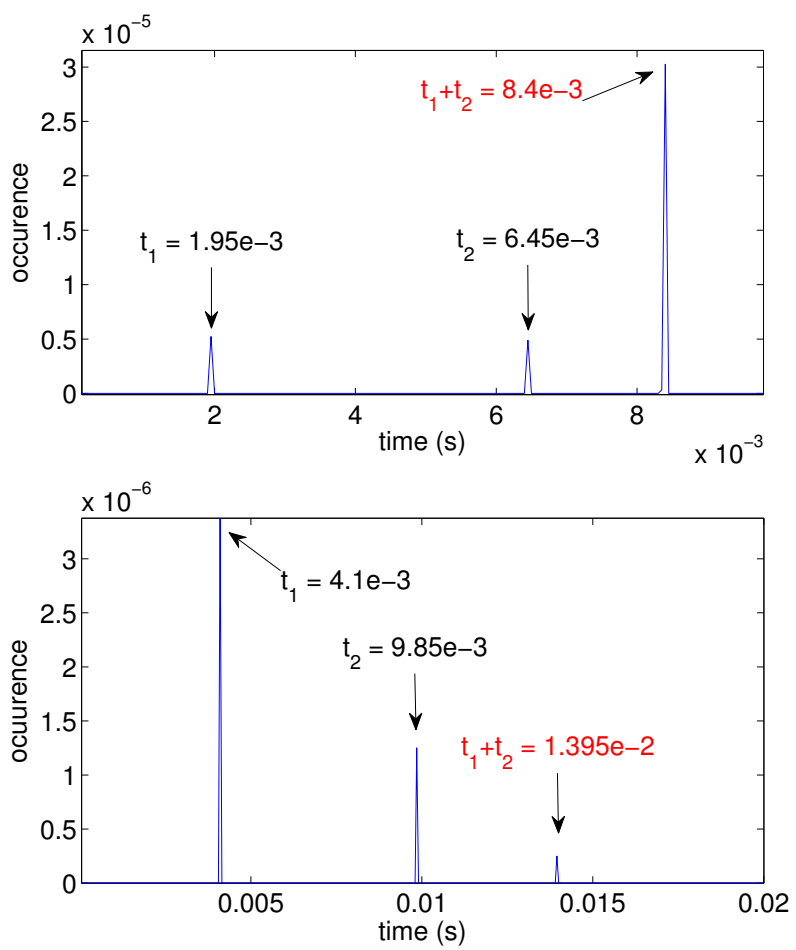

FIG. 8. Histograms of the return times associated with the multiphonics produced by an alto Zen On recorder with a $\mathrm{B}_{b}$ fingering (top) and a $\mathrm{B}$ fingering (bottom), for mouth pressure of 650 and $560 \mathrm{~Pa}$, respectively. The associated time series are represented in figure 4 .

developed in the last decades that allow to measure the input impedance of closed-open resonator, where the closed extremity corresponds to the exciter (musician) side [31]. These techniques rely on a sensor linked hermetically at the closed end of the resonator in place of the instrument exciter. Consequently, these are not suitable to determine experimentally the input admittance of open-open flutes resonators, which remains particularly challenging [32]. To the best of our knowledge, no precise measurement of the input admittance of a recorder has been performed. The case of pan-like flutes (which includes the Chilean flutes considered here) is again different, since the resonator is open on the exciter (musician) side and closed on the other side. Moreover, the face and the lips of the player (close to the open end) affect the radiation impedance [33]. For the case of the open-open resonator of the recorder, the numerical estimation of the input admittance using resonators models - implemented for example in the software WIAT [34] - is an alternative to experimental measurements. This requires the precise knowledge of the geometrical dimensions of the resonator, which are detailed in [15] for the alto Zen-On recorder (model Bressan) considered here.

Using this numerical approach, the input admittance of the considered recorder has been estimated for $\mathrm{B}_{b}$ and $\mathrm{B}$ fingerings (for which the multiphonics regimes are observed). The modulus 

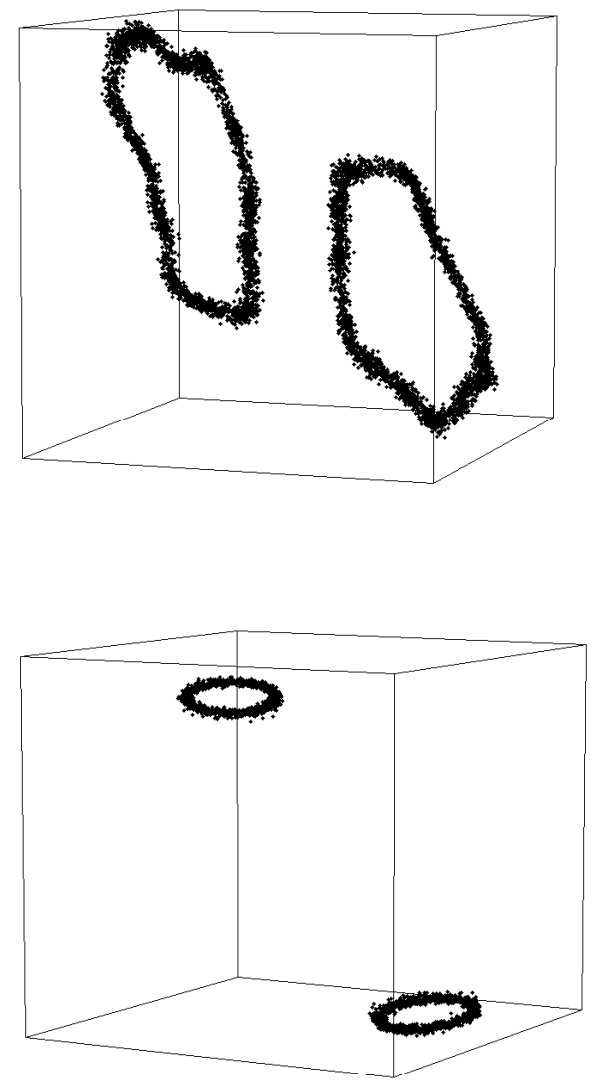

FIG. 9. Poincaré sections of the multiphonics represented in Figure 4, obtained with an alto Zen-On recorder for a $\mathrm{B}_{b}$ fingering (top) and for a $\mathrm{B}$ fingering (bottom). The recorder was played by a pressure-controlled artificial mouth, with mouth pressure of $650 \mathrm{~Pa}$ and $560 \mathrm{~Pa}$, respectively. The acoustic field was measured inside the resonator, under the labium.

of these input admittances are shown in Figure 10. These highlight, for each considered fingering, five resonance frequencies in the range [200;2000] Hz.

In parallel, we investigate further the spectra the two multiphonic regimes. These signals are measured with the artificial mouth and are thus stationary. Their Fourier analysis thus provides more insight than for sonidos rajados on the frequencies involved in the quasiperiodic oscillations. Figure 11 shows a zoom of the spectra already displayed in Figure 6. This highlights that all the peaks appear at frequencies than can be written as linear combinations of two base frequencies. More precisely, the multiphonic sound obtained with a $\mathrm{B}_{b}$ fingering has base frequencies $f_{b 1}=$ $477.1 \mathrm{~Hz}$ and $f_{b 2}=1551 \mathrm{~Hz}$, and the multiphonic played with a $\mathrm{B}$ fingering has base frequencies $f_{1}=500.2 \mathrm{~Hz}$ and $f_{2}=1217 \mathrm{~Hz}$.

A comparison between the base frequencies of the quasiperiodic regimes and the resonance frequencies of the instrument highlighted in Figure 10 shows that, for the $\mathrm{B}_{b}$ fingering, the base 
frequencies $f_{b 1}$ and $f_{b 2}$ of the quasiperiodic regime are close to the first and fourth resonance frequencies (peaks of the admittance) of the instrument, respectively. Similarly, the base frequencies $f_{1}$ and $f_{2}$ of the quasiperiodic regime obtained using the $\mathrm{B}$ fingering are close to the first and third resonance frequencies of the instrument, respectively. A more direct comparison between the resonance frequencies of the recorder for both fingerings considered here, and the base frequencies of the quasiperiodic regimes is provided in table I.

One can further observe that for both fingerings, the first resonance frequency of the instrument is (classically) close to the theoretical frequency of the notes usually played using these fingerings: $466 \mathrm{~Hz}$ for a $\mathrm{B}_{b}$ and $493 \mathrm{~Hz}$ for a $\mathrm{B}$. In both cases, the first register, defined as the periodic regime resulting from a coupling between the oscillating air jet and the first resonance frequency of the instrument, thus corresponds to the note usually seeked when the corresponding fingering is used by the musician. It is clear here that the base frequency $f_{b 1}$ is close to both the theoretical frequency of $a \mathrm{~B}_{b}$, and the first resonance frequency of the instrument with a $\mathrm{B}_{b}$ fingering. In the same way, the base frequency $f_{1}$ of the multiphonic obtained with a $\mathrm{B}$ fingering is close to the first resonance frequency of the instrument for this fingering, and differ from the theoretical frequency of a B by only 40 cents. This strongly suggests that for recorders, the quasiperiodic regimes result from an instability of the first register, induced by an higher resonance of the instrument, whose frequency is not commensurable with the oscillation frequency of the first register. Such an interpretation is consistent with the literature for flute-like instruments [5], but also for reed instruments. Indeed, the strong influence of the inharmonicity of the resonance frequencies of clarinet-like instruments on the generation of multiphonic regimes has been demonstrated both experimentally and numerically $[10,35]$. Moreover, this mechanism would explain the fact that $f_{1}$ and $f_{b 1}$ are 40 cents and 25 cents higher than the theoretical frequencies of a $\mathrm{B}$ and $\mathrm{a} \mathrm{B}_{b}$, respectively. Indeed, in both cases, the multiphonics regimes are experimentally observed for higher mouth pressure than the first register, and it is a well known characteristics of flute-like instruments that an increase of the mouth pressure leads to a substantial frequency shift towards the higher frequencies $[2,14]$.

\section{CONCLUSION}

We focused in this paper on non-periodic sounds produced by two different instruments of the flute family: two multiphonics produced by an alto recorder on the one hand, and the two sounds produced by two different flautas de chinos, called Catarra and Puntera, on the other hand. At first sight, multiphonics and sonidos rajados seem very different from each other: they can be easily 

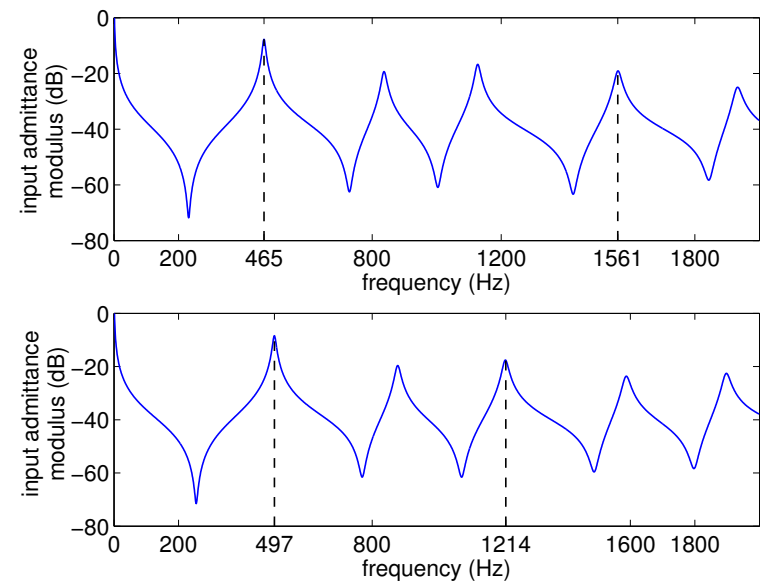

FIG. 10. Modulus of the input admittance of an alto Zen On recorder, for a $B_{b}$ fingering (top) and a $B$ fingering (bottom), computed from the geometrical dimensions of the resonator [15, 34]. The dark dotted lines indicate the resonance frequencies which are close to the base frequencies of the quasiperiodic regimes (see also table I).

\begin{tabular}{|c|c|c|}
\hline Fingering & $\begin{array}{c}\text { Instrument } \\
\text { resonance } \\
\text { frequencies }(\mathrm{Hz})\end{array}$ & $\begin{array}{c}\text { Multiphonics base } \\
\text { frequencies }(\mathrm{Hz})\end{array}$ \\
\hline & $\mathbf{4 6 5}$ & $f_{b 1}=\mathbf{4 7 7 . 1}$ \\
$\mathrm{B}_{b}$ & $\begin{array}{c}1127 \\
\end{array}$ & $f_{b 2}=\mathbf{1 5 5 1}$ \\
& $\mathbf{1 5 6 1}$ & $f_{1}=\mathbf{5 0 0 . 2}$ \\
$\mathrm{B}$ & $\mathbf{4 9 3 2}$ & $f_{2}=\mathbf{1 2 1 7}$ \\
& $\mathbf{4 9 1 9}$ & \\
& 1588 & \\
\hline
\end{tabular}

TABLE I. For an alto Zen-On recorder, comparison between the base frequencies of the multiphonics produced with $\mathrm{B}_{b}$ and $\mathrm{B}$ fingerings, and the first five resonance frequencies of the instrument for these fingerings.

distinguished from each other when listening, and both the deepness of the amplitude modulation and the frequency of this modulation are of different orders of magnitude. However, using numerical methods dedicated to the analysis of short and non-stationary time series, we demonstrated that despite their differences, both sonidos rajados and multiphonics are quasiperiodic regimes. To our knowledge, although both kinds of regimes have been investigated in the literature, neither their precise nature, nor the fact that they are similar regimes from a dynamical point of view, had been established before.

Moreover, it is usually considered that the production of sonidos rajados and multiphonics 

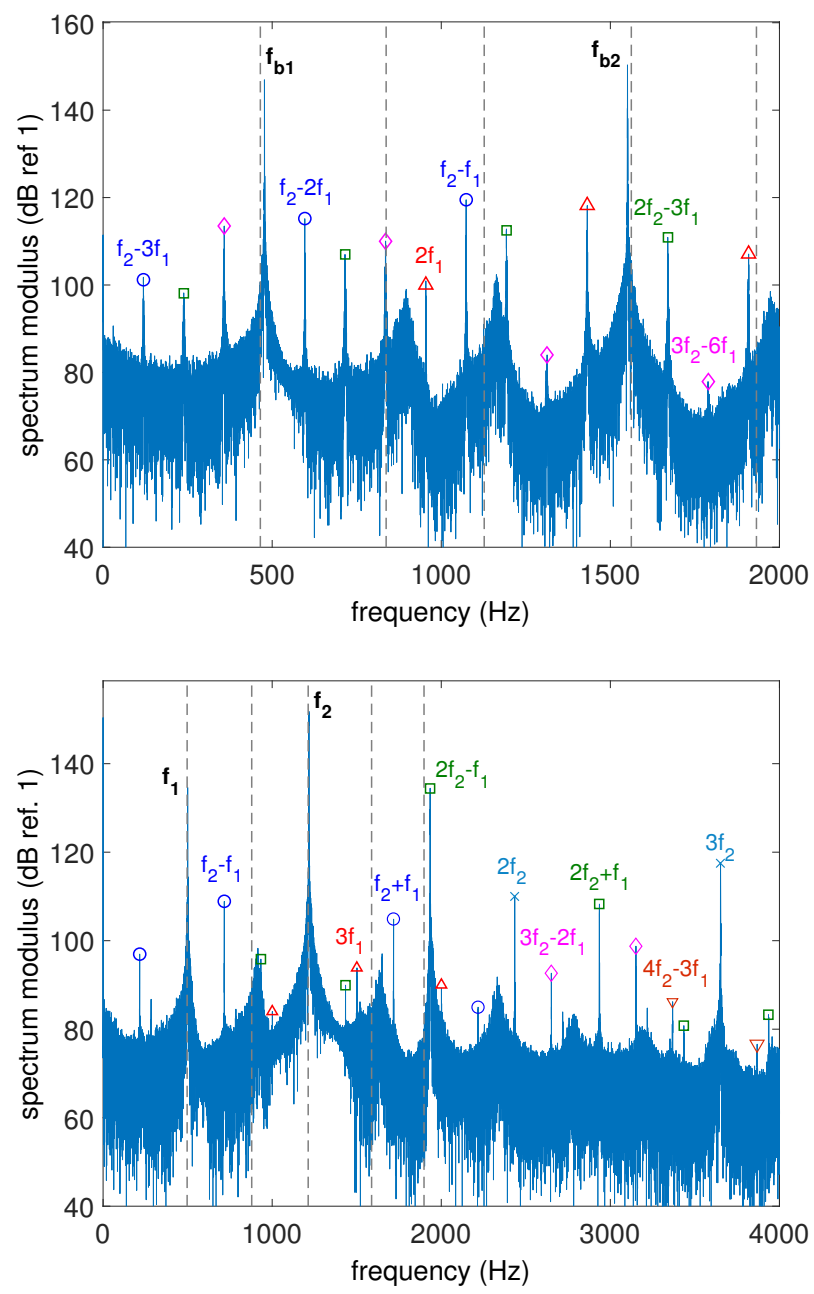

FIG. 11. Spectrum modulus of the internal acoustic field, measured under the labium of an alto Zen On recorder played by an artificial mouth, for two multiphonic sounds obtained with a $\mathrm{B}_{b}$ fingering (top) and a B fingering (bottom). The different peaks all occur at frequencies which are linear combinations of two base frequencies $f_{b 1}$ and $f_{b 2}$ for the $\mathrm{B}_{b}$ fingering (top), and $f_{1}$ and $f_{2}$ for the $\mathrm{B}$ fingering (bottom). Shown are the linear combinations $n \times f_{b 1}$ and $n \times f_{1}(\triangle), n \times f_{b 2}$ and $n \times f_{2}(\times), f_{b 2} \pm n \times f_{b 1}$ and $f_{2} \pm n \times f_{1}$ (॰), $2 f_{b 2} \pm n \times f_{b 1}$ and $2 f_{2} \pm n \times f_{1}(\square), 3 f_{b 2} \pm n \times f_{b 1}$ and $3 f_{2} \pm n \times f_{1}(\diamond), 4 f_{b 2} \pm n \times f_{b 1}$ and $4 f_{2} \pm n \times f_{1}$ $(\nabla)$, with $n$ a natural number. The dotted gray lines indicate the first five resonance frequencies of the instrument (as shown in Figure 10) for the considered fingerings.

results from different underlying physical phenomena [5]. However, their common quasiperiodic nature is a strong argument in favor of a common physical mechanism. In the case of the recorder, the comparison between the resonance frequencies of the instrument and the base frequencies of the quasiperiodic regimes suggests that the quasiperiodic oscillations result from an instability of the first register, induced by an higher resonance frequency of the instrument. This instability appears when the blowing pressure in the musician's mouth is sufficiently high.

In order to further investigate these phenomena and to understand the production mechanism 
of quasiperiodic sounds in both flautas de chinos and recorders, an in-depth bifurcation analysis of a physical model of the instrument would be particularly valuable. This would allow for a more comprehensive comparison between the two types of sounds, which could explain the observed differences, for example in terms of amplitude and frequency of the modulation. Moreover, this would allow to further investigate the influence of the making parameters of the instruments on the emergence of quasiperiodic sounds. Our preliminary results suggest that studying the influence of the input admittance of the instrument (inharmonicity, relative amplitude of the different resonance peaks) would be of particular interest. Quasiperiodic sounds are either considered as a suitable behaviour or as a defect of the instruments. Therefore, understanding how to trigger or to avoid these regimes would be particularly valuable in the context of instrument making.

\section{ACKNOWLEDGEMENTS}

This work is supported by CONICYT through FONDECYT Project No1201551. 
[1] N. H. Fletcher and L. M. Douglas, The Journal of the Acoustical Society of America 68, 767 (1980).

[2] J. W. Coltman, The Journal of the Acoustical Society of America 40, 99 (1966).

[3] R. Auvray, B. Fabre, and P.-Y. Lagrée, The Journal of the Acoustical Society of America 131, 1574 (2012).

[4] M. Castellengo, 34 (1982).

[5] J. W. Coltman, The Journal of the Acoustical Society of America 120, 2312 (2006).

[6] M. Castellengo and S. Chantreuil, in Comptes-Rendus du 11ème I.C.A. (1983).

[7] M. Castellengo, Bulletin du groupe d'acoustique musicale 74 (1974).

[8] J. P. De Arce, The Galpin Society Journal 51, 17 (1998).

[9] J. Backus, The Journal of the Acoustical Society of America 63, 591 (1978).

[10] J.-B. Doc, C. Vergez, and S. Missoum, Acta Acustica united with Acustica 100, 543 (2014).

[11] F. Blanc, P. De La Cuadra, B. Fabre, G. Castillo, and C. Vergez, in Proc. of 20th Int. Symposium on Music Acousitcs (2010).

[12] P. Bolton, "Recorder maker," personal communication.

[13] H. Wright and D. Campbell, The Galpin Society Journal 51, 51 (1998).

[14] N. H. Fletcher, The Journal of the Acoustical Society of America 60, 926 (1976).

[15] D. H. Lyons, The Journal of the Acoustical Society of America 70, 1239 (1981).

[16] A. Chaigne and J. Kergomard, Acoustics of musical instruments (Springer, 2016).

[17] D. Ferrand, C. Vergez, B. Fabre, and F. Blanc, Acta Acustica united with Acustica 96, 701 (2010).

[18] S. Terrien, R. Blandin, C. Vergez, and B. Fabre, Acta Acustica united with Acustica 101, 300 (2015).

[19] See supplementary material at URL to be included for the corresponding sounds.

[20] P. De la Cuadra, "Personal webpage," https://ccrma.stanford.edu/ pdelac/Antaras/html/ Pucalan_Puntera.html, accessed: 2020-12-01.

[21] See supplementary material at URL to be included for the corresponding sounds.

[22] H. Abarbanel, Analysis of observed chaotic data (Springer Science \& Business Media, 2012).

[23] J. Guckenheimer and P. Holmes, Nonlinear oscillations, dynamical systems, and bifurcations of vector fields, Vol. 42 (Springer Science \& Business Media, 2013).

[24] Y. A. Kuznetsov, Elements of applied bifurcation theory, Vol. 112 (Springer Science \& Business Media, 2013).

[25] J. Eckmann, S. O. Kamphorst, D. Ruelle, et al., World Scientific Series on Nonlinear Science Series A 16, 441 (1995).

[26] L. Cao, Physica D: Nonlinear Phenomena 110, 43 (1997).

[27] Y. Zou, Exploring recurrences in quasiperiodic systems, Ph.D. thesis, University of Potsdam (2007).

[28] C. Merkwirth, U. Parlitz, D. Wedekind, I Engster, and W. Lauterborn, OpenTSTOOL user manual, Tech. Rep. (Gttingen, Germany: Drittes Physikalisches Institut, Universitt Gttingen, 2009). 
[29] B. Balachandran, T. Kalmár-Nagy, and D. E. Gilsinn, Delay differential equations (Springer, 2009).

[30] A. Ernoult and B. Fabre, Acta Acustica united with Acustica 103, 106 (2017).

[31] J.-P. Dalmont, Journal of Sound and Vibration 243, 427 (2001).

[32] J. Wolfe, J. Smith, J. Tann, and N. H. Fletcher, Journal of sound and vibration 243, 127 (2001).

[33] A. Ernoult, P. De La Cuadra, and B. Fabre, Acta Acustica united with Acustica 104, 496 (2018).

[34] A. Lefebvre, "The wind instrument acoustic toolkit," http://www.music.mcgill.ca/caml/doku.php? id=wiat : wiat.

[35] J.-B. Doc and C. Vergez, The Journal of the Acoustical Society of America 137, 1756 (2015). 